\title{
Nonlocal response in plasmonic waveguiding with extreme light confinement
}

Toscano, Giuseppe; Raza, Søren; Yan, Wei; Jeppesen, Claus; Xiao, Sanshui ; Wubs, Martijn; Jauho, Antti-Pekka; Bozhevolnyi, Sergey I.; Mortensen, N. Asger

Published in:

Nanophotonics

Link to article, DOI:

10.1515/nanoph-2013-0014

Publication date:

2013

Document Version

Publisher's PDF, also known as Version of record

Link back to DTU Orbit

Citation (APA):

Toscano, G., Raza, S., Yan, W., Jeppesen, C., Xiao, S., Wubs, M., Jauho, A-P., Bozhevolnyi, S. I., \& Mortensen, N. A. (2013). Nonlocal response in plasmonic waveguiding with extreme light confinement. Nanophotonics, 2(3), 161-166. https://doi.org/10.1515/nanoph-2013-0014

\section{General rights}

Copyright and moral rights for the publications made accessible in the public portal are retained by the authors and/or other copyright owners and it is a condition of accessing publications that users recognise and abide by the legal requirements associated with these rights.

- Users may download and print one copy of any publication from the public portal for the purpose of private study or research.

- You may not further distribute the material or use it for any profit-making activity or commercial gain

- You may freely distribute the URL identifying the publication in the public portal

If you believe that this document breaches copyright please contact us providing details, and we will remove access to the work immediately and investigate your claim. 


\title{
Regular article
}

\author{
Giuseppe Toscano, Søren Raza, Wei Yan, Claus Jeppesen, Sanshui Xiao, Martijn Wubs, \\ Antti-Pekka Jauho, Sergey I. Bozhevolnyi and N. Asger Mortensen*
}

\section{Nonlocal response in plasmonic waveguiding with extreme light confinement}

\begin{abstract}
We present a novel wave equation for linearized plasmonic response, obtained by combining the coupled real-space differential equations for the electric field and current density. Nonlocal dynamics are fully accounted for, and the formulation is very well suited for numerical implementation, allowing us to study waveguides with subnanometer cross-sections exhibiting extreme light confinement. We show that groove and wedge waveguides have a fundamental lower limit in their mode confinement, only captured by the nonlocal theory. The limitation translates into an upper limit for the corresponding Purcell factors, and thus has important implications for quantum plasmonics.
\end{abstract}

Keywords: Nanoplasmonics; nonlocal response; light confinement; waveguides; light-matter interactions.

PACS numbers: 78.67.Uh; 78.67.Lt; 71.45.Lr; 73.20.Mf; 41.20.Jb.

\footnotetext{
*Corresponding author: N. Asger Mortensen, Department of Photonics Engineering, Technical University of Denmark, DK-2800 Kgs. Lyngby, Denmark; and Center for Nanostructured Graphene (CNG), Technical University of Denmark, DK-2800 Kgs. Lyngby, Denmark, e-mail: asger@mailaps.org

Giuseppe Toscano and Claus Jeppesen: Department of Photonics Engineering, Technical University of Denmark, DK-2800 Kgs. Lyngby, Denmark

Søren Raza: Department of Photonics Engineering, Technical University of Denmark, DK-2800 Kgs. Lyngby, Denmark; and Center for Electron Nanoscopy, Technical University of Denmark, DK-2800 Kgs. Lyngby, Denmark

Wei Yan, Sanshui Xiao and Martijn Wubs: Department of Photonics Engineering, Technical University of Denmark, DK-2800 Kgs. Lyngby, Denmark; and Center for Nanostructured Graphene (CNG), Technical University of Denmark, DK-2800 Kgs. Lyngby, Denmark Antti-Pekka Jauho: Center for Nanostructured Graphene (CNG), Technical University of Denmark, DK-2800 Kgs. Lyngby, Denmark; and Department of Micro and Nanotechnology, Technical University of Denmark, DK-2800 Kgs. Lyngby, Denmark

Sergey I. Bozhevolnyi: Institute of Technology and Innovation, University of Southern Denmark, DK-5230 Odense, Denmark
}

Edited by Jennifer A. Dionne
Wave propagation along dielectric waveguide structures has over the years been extended also to plasmonic systems with waveguide modes in the form of surfaceplasmon polaritons. Plasmonic waveguides have attracted considerable attention during the past decade, primarily due to their ability to support extremely confined modes, i.e., modes that do not exhibit a diffraction-limited cutoff for progressively smaller waveguide cross sections but transform themselves into their electrostatic counterparts [1]. Investigations of nanowire [2], groove [3] and wedge [4] waveguides, shown to ensure extreme light confinement, raise a natural interest in the influence of nonlocal effects on strongly confined plasmonic modes [5]. Waveguiding by metal nanowires [6] and more recently plasmonic focusing by conical tips $[7,8]$ have been studied in the context of nonlocal response. However, with the exception of few analytical studies of simple planar geometries $[9,10]$, nonlocal effects in the dispersion properties of complex waveguides remain unexplored, a circumstance that can partly be explained by the added complexity due to nonlocal effects as compared to the widespread framework of the local-response approximation (LRA) [11].

There is also another good reason to look for nonlocal effects in extreme light confinement. Subwavelength mode confinement implies large effective Purcell factors and thereby strong coupling of single emitters to nearby plasmonic waveguide modes [12]. The latter opens a doorway to quantum optics with surface plasmons, including the possibilities for realization of single-photon transistors [13] and long-distance entanglement of qubits [14]. Since one would expect that the plasmonic mode confinement is fundamentally limited by nonlocal effects, similarly to nonlocal limits in the field enhancement of localized plasmon excitations $[15,16]$, studies of the plasmonic mode confinement beyond the LRA are of great interest for quantum plasmonics. More specifically, in the LRA higher single-photon efficiencies [12] and Purcell factors [13] have been found to occur for smaller waveguide radii $R$, and the $R \rightarrow 0$ limit is commonly taken to estimate the strongest light-matter interactions. Nonlocal response effects 
become increasingly important in this $R \rightarrow 0$ limit, which is an important motivation for our present study of nonlocal effects for highly confined plasmonic waveguides.

In this Letter, we derive a novel wave equation which fully takes into account the nonlocal dynamics of an oftenemployed hydrodynamical model (HDM). We apply the wave equation to plasmonic waveguides (Figure 1) with extreme light confinement, defined by the subnanometer dimensions of the waveguide cross section. After stringent bench-marking of our approach against the analytically tractable case of nanowires with circular cross-section, we analyze in detail groove and wedge waveguides and demonstrate the existence of fundamental limits in their mode confinement and Purcell factors, imposed by the nonlocal effects. At the same time, our results reveal that there is room for downsizing present-day quantum plasmonic devices before these fundamental limitations set in.

The nonlocal response, or spatial dispersion, is a consequence of the quantum many-body properties of the electron gas, which we here take into account within a semi-classical model [17-20]. In this model the equationof-motion for an electron in an electrical field is supplemented with a hydrodynamic pressure term originating from the quantum kinetics of the electron gas. By linearization, the plasmonic response is governed by the following pair of coupled real-space differential equations [21]:

$$
\begin{gathered}
\nabla \times \nabla \times \mathbf{E}(\mathbf{r})=\left(\frac{\omega}{c}\right)^{2} \mathbf{E}(\mathbf{r})+i \omega \mu_{0} \mathbf{J}(\mathbf{r}), \\
\frac{\beta^{2}}{\omega(\omega+i / \tau)} \nabla[\nabla \cdot \mathbf{J}(\mathbf{r})]+\mathbf{J}(\mathbf{r})=\sigma(\mathbf{r}) \mathbf{E}(\mathbf{r}) .
\end{gathered}
$$

Here, the term $\nabla[\nabla \cdot \mathbf{J}]=\nabla \times \nabla \times \mathbf{J}+\nabla^{2} \mathbf{J}$ is a correction to Ohm's law and scales as $\beta^{2}=(3 / 5) v_{F}^{2}$ within the ThomasFermi model [22] with $v_{F}$ being the Fermi velocity. For simplicity we neglect here any interband effects present in

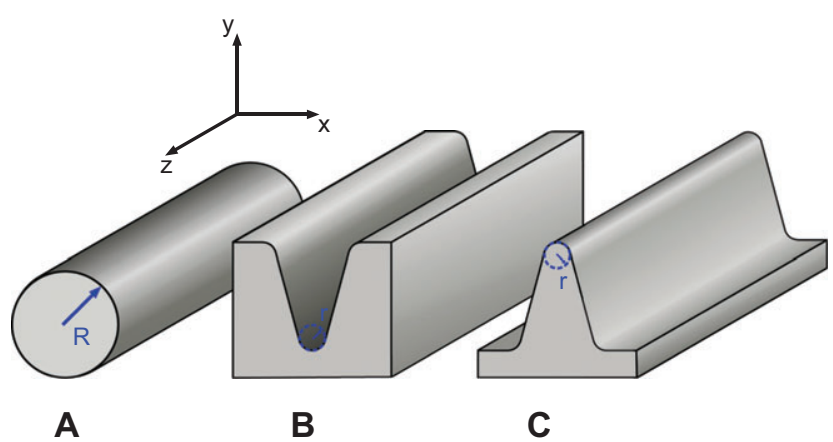

Figure 1 Generic plasmonic waveguiding geometries with wave propagation in the $z$-direction and extreme transverse confinement in the $x y$-plane due to subnanometer geometric dimensions, e.g., the nanowire radius $R$ or the edge radius-of-curvature $r$. real metals; these can be included straightforwardly [23, see Supplemental material]. In our numerical solutions we will consider Drude parameters appropriate for silver [24]. Assuming a hard-wall confinement associated with a high work function, the boundary conditions for the current at the metal surface become particularly simple: the tangential component is unrestricted while the normal component vanishes due to the current continuity and vanishing of all electron wave functions at the surface [10, 21].

For analytical progress one can eliminate the current from Eq. (1a), thereby arriving at an integral equation where a dyadic Green's function accounts for the nonlocal dynamics of the electron gas $[25,26]$. Alternatively, the coupled equations (1a) and (1b) form a natural starting point for a numerical treatment of arbitrarily shaped metallic nanostructures, e.g., with a state-of-the-art finite-element method [23, 27]. Recently, we employed this approach to study field enhancement and SERS in groove structures [15]. However, for waveguiding geometries we seek solutions of the form $\mathbf{E}(\mathbf{r}) \propto \exp \left(i k_{z} z\right)$ leading to an eigenvalue problem for $k_{z}(\omega)$ with a six-component eigenvector $\{\mathbf{E}, \mathbf{J}\}$. In that context the coupled-equation formulation is numerically less attractive. Here, instead, we eliminate the current from Eq. (1b), a procedure that, after straightforward manipulations using standard vector calculus (see Supplemental material), results in an appealingly compact, but yet entirely general nonlocal wave equation:

$$
\begin{aligned}
& \nabla \times \nabla \times \mathbf{E}(\mathbf{r})=\left(\frac{\omega}{c}\right)^{2} \hat{\varepsilon}_{\mathrm{NL}}(\mathbf{r}) \mathbf{E}(\mathbf{r}), \\
& \hat{\varepsilon}_{\mathrm{NL}}(\mathbf{r})=\varepsilon_{D}(\mathbf{r})+\frac{\beta^{2}}{\omega(\omega+i / \tau)} \nabla^{2} .
\end{aligned}
$$

Here, the operator $\hat{\varepsilon}_{\mathrm{vN}}(\mathbf{r})$ contains the nonlocal effects. In the limit $\beta \rightarrow 0, \hat{\varepsilon}_{\mathrm{NL}}(\mathbf{r})$ reduces to the usual Drude dielectric function $\varepsilon_{D}(\mathbf{r})=1+i \sigma(\mathbf{r}) /\left(\varepsilon_{0} \omega\right)=1-\omega_{p}^{2}(\mathbf{r}) /[\omega(\omega+i / \tau)]$ used in the LRA. Thus, with a simple rewriting we have turned the coupled-wave equations into a form reminiscent of the usual wave equation, with all aspects of nonlocal response contained in the Laplacian term $\beta^{2} \nabla^{2}$ in $\hat{\varepsilon}_{\mathrm{NL}}(\mathbf{r})$. This is the main theoretical result of this Letter. In passing, we note that with Eq. (2b) we immediately recover the dispersion relation $\omega(k)=\sqrt{\omega_{p}^{2}+\beta^{2} k^{2}}$ for bulk plasmons in translationally invariant plasma (see Supplemental material). Clearly, the single-line form is beneficial for the conceptual understanding and further analytical progress, as well as for numerical implementations: the additional Laplacian does not add any complications beyond those already posted by the double-curl operator on the left-hand side equation. Likewise the boundary condition that was imposed on the current $\mathbf{J}$ in Eq. (1) 
translates into an additional boundary condition on the electric field in Eq. (2), see (Supplemental material). While Eq. (1) can be solved numerically for scattering problems $[15,23,27]$ and some waveguide problems [28], the result in Eq. (2) is clearly a major advancement for efficient and accurate numerical eigenvalue solutions in waveguiding geometries with arbitrarily shaped waveguide cross sections. In particular, differential operations reduce to a Laplacian and the dimension of the eigenvalue problem is reduced from six field components to only three.

We now apply the developed formalism to the waveguide configurations of Figure 1 which can provide extreme light confinement [1]: i) metal nanowires with circular cross sections [2] where analytical solutions [7] are available for benchmarking of the numerics, ii) grooves in metal [3], and iii) metal wedges [4]. In addition to the usual mode characteristics, effective index and propagation length, we also evaluate the effective mode area: $A_{\text {eff }}=V_{\text {eff }} / L$, where $V_{\text {eff }}$ is the effective mode volume associated with the Purcell effect, i.e.,

$$
A_{\text {eff }}=\frac{\int_{V_{m}+V_{a}} \mathrm{~d} x \mathrm{~d} y u(\mathbf{r})}{\max _{V_{a}}\{u(\mathbf{r})\}},
$$

where $u(\mathbf{r})$ is the electromagnetic energy functional (see Supplemental material). The cross-sectional integral extends over the volumes $V_{m}$ and $V_{a}$ occupied by metal and air, respectively, while the evaluation of the maximal energy density is restricted to the air region where dipole emitters can be placed.

The dispersion curves and effective mode areas (normalized to the nanowire cross section) calculated for silver nanowires of different radii (Figure 2A,B) exhibit a blueshift and increased mode area (for fixed $k_{z}$ ) when taking nonlocal effects into account. The numerical results of Eq. (2) show excellent agreement with the corresponding analytical results previously derived from Eq. (1) [7]. Importantly, nonlocal dynamics influences strongly the mode field distribution (see Figure 2C), because, contrary to the LRA case, the normal component of the electrical field within the HDM is continuous across the interfaces (this is a special case for a Drude metal without interband effects and surrounded by vacuum [See Supplemental material]). It is indeed seen (Figure $2 \mathrm{C}$ ) that $\left|\mathbf{E}_{\rho}\right|$ is discontinuous on the boundary in the local case, while it varies continuously across the boundary in the nonlocal case. This variation occurs in a region extending over $\approx 0.1 \mathrm{~nm}$, that is of the order of the Fermi wavelength of silver.

The results for cylindrical nanowires, while demonstrating the main effects of nonlocal dynamics on the mode characteristics, indicate that the quantitative

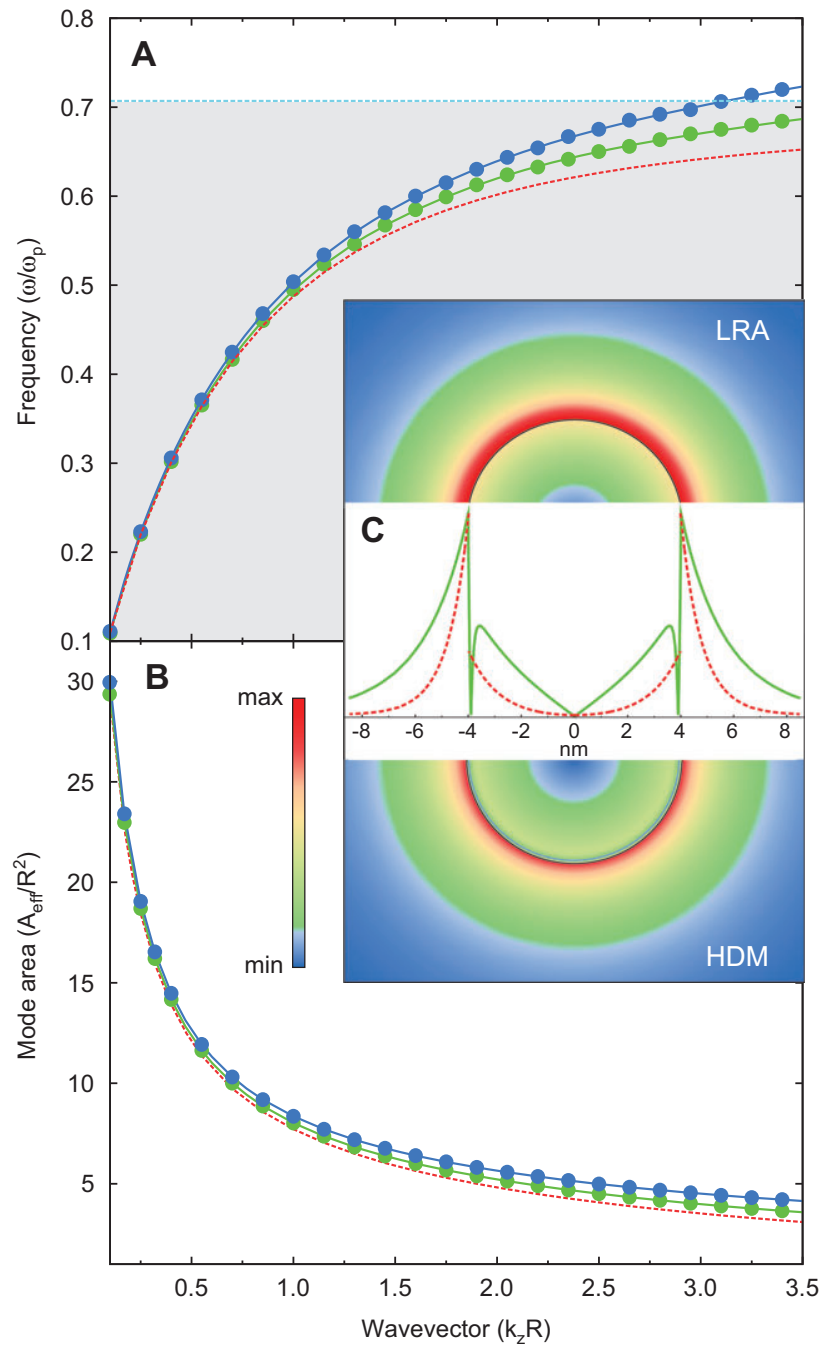

Figure 2 Fundamental waveguide mode of a cylindrical silver nanowire embedded in air. (A) Dispersion relation $\omega\left(k_{z}\right)$ and (B) normalized effective mode area within the HDM for the nanowire radius $R=2 \mathrm{~nm}$ (blue) and $4 \mathrm{~nm}$ (green), respectively, showing excellent agreement between numerical solutions of Eq. (2) (solid points) and analytical results (solid lines). For comparison, the red-dashed curve shows the universal result of the nonretarded LRA, with its large- $k_{z}$ limiting value of $\omega_{p} / \sqrt{2}$ indicated in (A) by the horizontal line. (C) Radial distribution of the electric field $\left|\mathbf{E}_{\rho}\right|$ at $\omega=0.6 \omega_{\mathrm{p}}$ for $R=4 \mathrm{~nm}$, contrasting the continuous field variation in the HDM with its usual boundary discontinuity in the LRA.

changes are modest even for very small radii (Figure 2). In order to explore fundamental limitations, one has to consider the limit of vanishing radii of curvature. While subnanometer radii appear unrealistic for nanowires, fabrication of grooves cut in metal and metal wedges, e.g., by nanoimprint lithography [29], can in fact result in $\mathrm{nm}$ sharp edges with corresponding $\mathrm{nm}$-sized wedge modes [4]. We expect that nonlocal effects then come into play.

Rather surprisingly, the mode effective index and propagation length calculated for silver grooves and 
wedges (Figure 3) exhibit even weaker influence of the nonlocal effects as compared to the case of nanowires (Figure 2). In fact, there is no noticeable difference between the LRA- and HDM-based results obtained for 1-nm-radius of edges. In the limit of mathematically sharp edges, the mode effective index becomes only slightly larger and the propagation length slightly smaller than those calculated for $1 \mathrm{~nm}$ edge radius (Figure 3). We explain this result by the fact that groove and wedge plasmonic modes are only

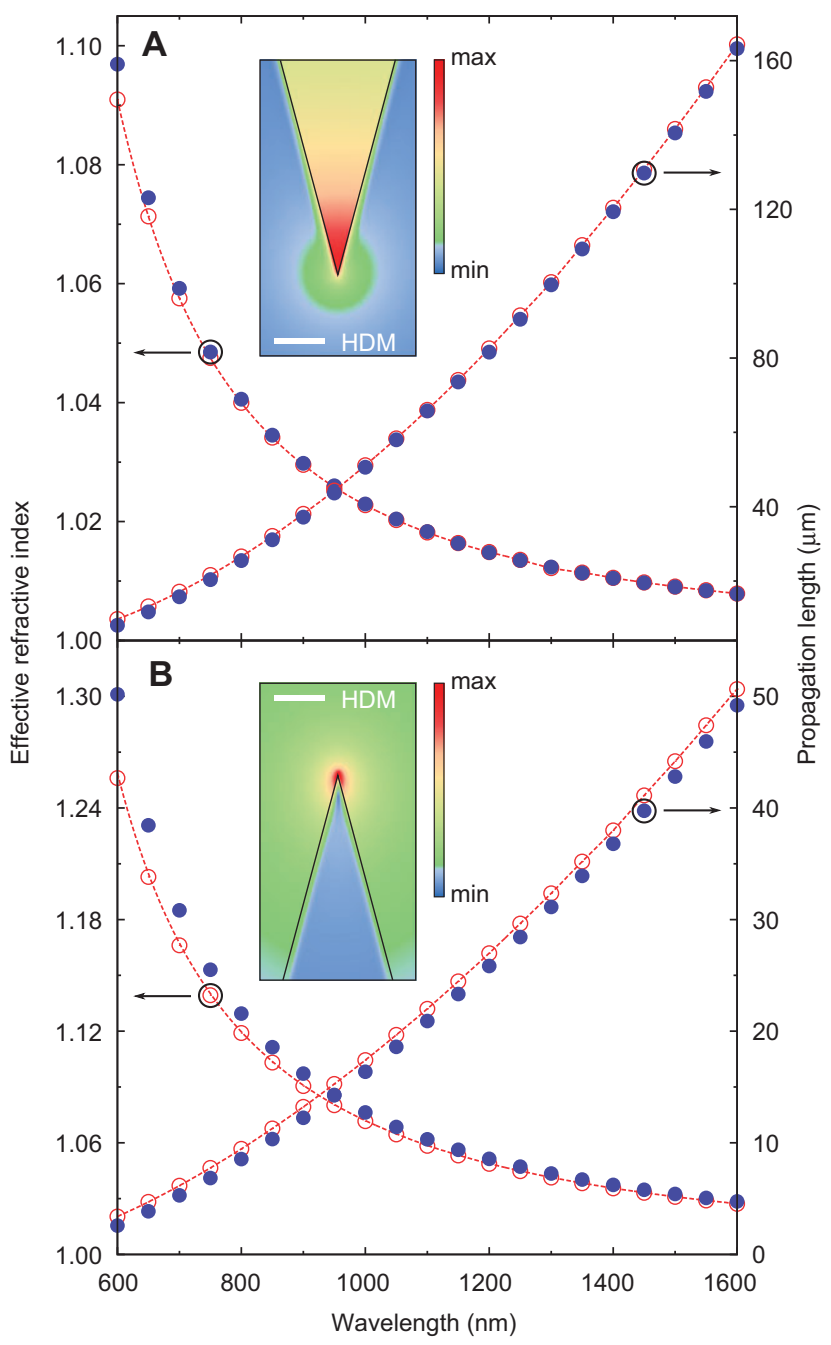

Figure 3 Effective index (left axis) and propagation length (right axis) versus wavelength for the fundamental mode in complimentary (A) V-groove and (B) wedge silver waveguides, both with an opening angle of $30^{\circ}$. The nonlocal results (solid circular symbols) obtained with Eq. (2) are contrasted to the LRA (open circles), with dashed lines serving as eye guides. Results for mathematically sharp structures with $r=0$ (blue solid circles) are contrasted to finite rounding with $r=1 \mathrm{~nm}$ (red open circles). Insets show field-intensity distributions (white scale bars are $1 \mathrm{~nm}$ long) calculated within the HDM $(\lambda=600$ $\mathrm{nm}$ ) for infinitely sharp edges. The fingerprint of nonlocal effects is clearly visible as the field penetrates into the metal by a distance of the order of the Fermi wavelength of silver. partially affected by the very tip, being distributed also and predominantly over flat edges (see insets in Figure 3).

The situation changes drastically when one considers the mode confinement, using the mode area associated with the Purcell factor, Eq. (3). We recall that the field enhancement calculated within the LRA grows without bound for progressively sharper pointed structures while it remains finite when calculated within HDM $[15,16]$. Analogously, in the present case, one may expect that the mode area calculated within the LRA decreases without bound for a decreasing edge radius, while it may

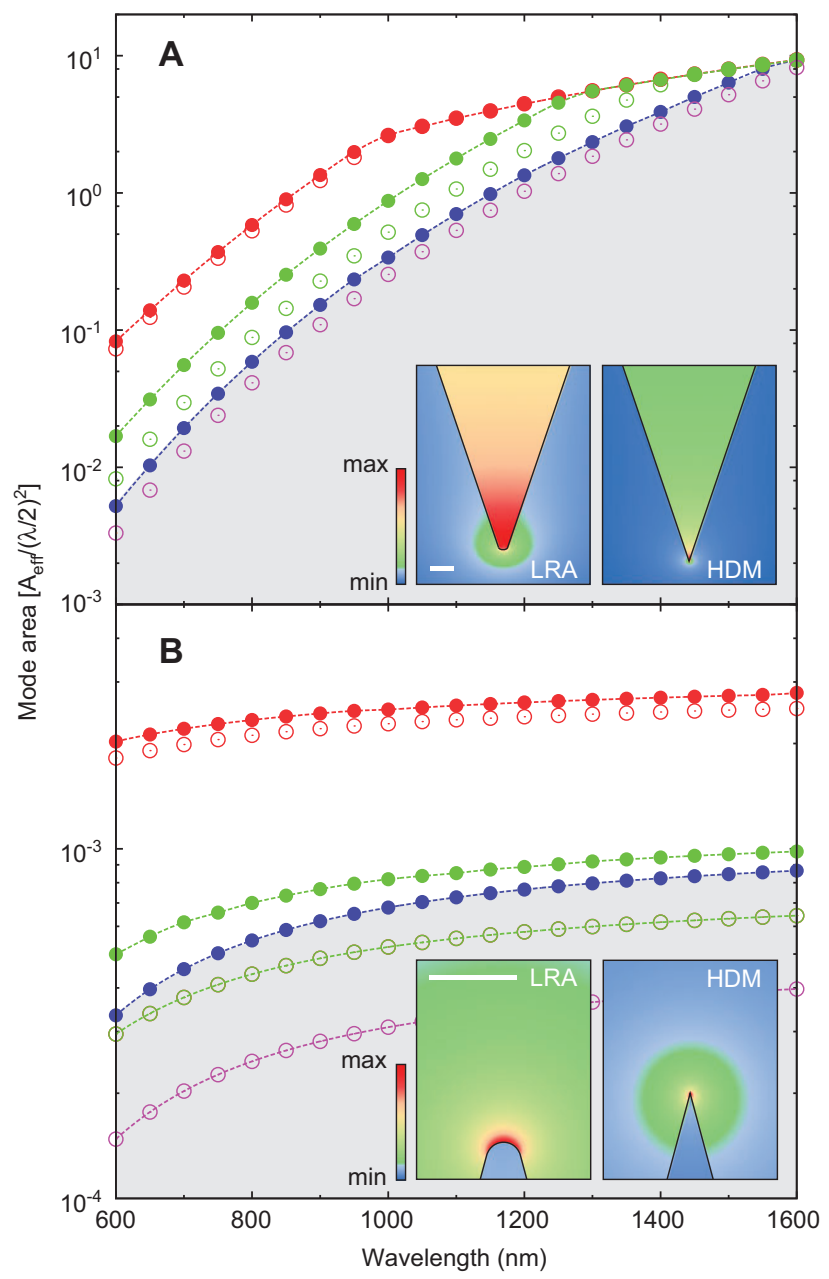

Figure 4 Normalized mode area versus wavelength for the fundamental mode in complimentary (A) V-groove and (B) wedge silver waveguides, both with opening angles of $30^{\circ}$. The HDM results (solid symbols) are contrasted to the LRA (open circles) for $r=1 \mathrm{~nm}$ (red) and $r=0.2 \mathrm{~nm}$ (green). Results for mathematically sharp structures with $r=0$ (blue solid circles) define a lower limit in the HDM (gray-shaded regions are inaccessible). For the LRA, the $r=0.1 \mathrm{~nm}$ results (magenta) exceed this limit and the mode area tends to zero when $r \rightarrow 0$. Insets show field-intensity distributions (white scale bars are $5 \mathrm{~nm}$ long) at $\lambda=600 \mathrm{~nm}$. The LRA intensities are with rounding $r=1 \mathrm{~nm}$, while $r=0$ is used for the HDM maps. 
saturate within the HDM. LRA-based simulations for subnanometer radii of edges show (Figure 4) that the mode area indeed tends to zero, without any apparent saturation. This trend is more pronounced for wedges than for grooves, because the wedge geometry ensures generally a better mode confinement (cf. Figure 4A and B), as was also noted previously [4]. At the same time, the simulations conducted within the HDM demonstrate clearly the existence of a lower bound for the mode area which remains finite even for mathematically sharp edges (blue circles in Figure 4). The associated Purcell factors can be estimated by inverse of the normalized mode areas displayed in Figure 4 [30]. Thus, our calculations show that there is a fundamental limit for the maximum Purcell factors achievable with plasmonic waveguides. It is interesting that the upper limit of Purcell factors evaluated in this way decreases noticeably in the long-wavelength regime. This feature is related to a general weakening of all plasmonic effects, including waveguiding [1], for longer wavelengths (with metals approaching the limiting case of perfect conductors). At the same time, in the case of wedges, these factors remain substantial even at telecom wavelengths, with the propagation lengths becoming considerably long (Figure 3 ) and amenable for circuitry application. It should also be borne in mind that the plasmonic field confinement in both grooves and wedges increases for smaller opening angles [3, 4], so that even larger Purcell factors can be achieved, albeit at the expense of shorter propagation.

In conclusion, using a novel wave equation accounting for nonlocal dynamics, we considered plasmonic waveguides with extreme light confinement and demonstrated the existence of a fundamental limit in their mode confinement imposed by nonlocal effects. Our results imply fundamental limitations in the corresponding Purcell factors, showing at the same time the possibility of achieving very high Purcell factors with V-groove and wedge waveguides that ensure sufficiently long propagation lengths for applications in quantum plasmonics. Here, we have focused on single-connected metal geometries where dominating currents are naturally of an Ohmic nature, whereas tunneling currents may cause important limitations too in e.g., closely spaced metallic objects [31]. Finally, beyond the linear response fundamental limitations may arise due to nonlinearities [32].

Acknowledgments: This work was financially supported by an H.C. Ørsted Fellowship (W.Y.) and the Center for Nanostructured Graphene (CNG) is sponsored by the Danish National Research Foundation, Project DNRF58.

Received May 15, 2013; revised June 19, 2013; accepted June 19, 2013

\section{References}

[1] Gramotnev DK, Bozhevolnyi SI. Plasmonics beyond the diffraction limit. Nat Photonics 2010;4:83-91.

[2] Ditlbacher H, Hohenau A, Wagner D, Kreibig U, Rogers M, Hofer F, Aussenegg FR, Krenn JR. Silver nanowires as surface plasmon resonators. Phys Rev Lett 2005;95:257403.

[3] Bozhevolnyi SI, Volkov VS, Devaux E, Ebbesen TW. Channel plasmon-polariton guiding by subwavelength metal grooves. Phys Rev Lett 2005;95:046802.

[4] Moreno E, Rodrigo SG, Bozhevolnyi SI, Martín-Moreno L, García-Vidal FJ. Guiding and focusing of electromagnetic fields with wedge plasmon polaritons. Phys Rev Lett 2008;100:023901.

[5] García de Abajo FJ. Nonlocal effects in the plasmons of strongly interacting nanoparticles, dimers, and waveguides. J Phys Chem C 2008;112:17983-7.

[6] Aers GC, Boardman AD, Paranjape BV. Non-radiative surface plasma-polariton modes of inhomogeneous metal circular cylinders. J Phys F: Metal Phys 1980;10:53.

[7] Ruppin R. Effect of non-locality on nanofocusing of surface plasmon field intensity in a conical tip. Phys Lett $A$ 2005;340:299-302.

[8] Wiener A, Fernández-Domínguez AI, Horsfield AP, Pendry JB, Maier SA. Nonlocal effects in the nanofocusing performance of plasmonic tips. Nano Lett 2012;12:3308-14.
[9] Boardman AD, Paranjape BV, Nakamura YO. Surface plasmonpolaritons in a spatially dispersive inhomogeneous medium. Phys Stat Sol B 1976;75:347-59.

[10] Yan W, Wubs M, Mortensen NA. Hyperbolic metamaterials: nonlocal response regularizes broadband supersingularity. Phys Rev B 2012;86:205429.

[11] Maier SA. Plasmonics: fundamentals and applications. New York: Springer, 2007.

[12] Chang DE, Sørensen AS, Hemmer PR, Lukin MD. Quantum optics with surface plasmons. Phys Rev Lett 2006;97: 053002.

[13] Chang DE, Sørensen AS, Demler EA, Lukin MD. A single-photon transistor using nanoscale surface plasmons. Nat Phys 2007;3:807-12.

[14] González-Tudela A, Martín-Cano D, Moreno E, Martín-Moreno L, Tejedor C, García-Vidal FJ. Entanglement of two qubits mediated by one-dimensional plasmonic waveguides. Phys Rev Lett 2011;106:020501.

[15] Toscano G, Raza S, Xiao S, Wubs M, Jauho A-P, Bozhevolnyi SI, Mortensen NA. Surface-enhanced Raman spectroscopy: nonlocal limitations. Opt Lett 2012;37:2538-40.

[16] Ciracì C, Hill RT, Mock JJ, Urzhumov Y, Fernández-Domínguez Al, Maier SA, Pendry JB, Chilkoti A, Smith DR. Probing the ultimate limits of plasmonic enhancement. Science 2012;337:1072-4. 
[17] Bloch F. Stopping power of atoms with several electrons. Z Phys A 1933;81:363.

[18] Barton G. Some surface effects in the hydrodynamic model of metals. Rep Prog Phys 1979;42:963.

[19] Boardman A. Electromagnetic surface modes. Hydrodynamic theory of plasmon-polaritons on plane surfaces. Chichester: John Wiley and Sons, 1982.

[20] Pitarke J, Silkin V, Chulkov E, Echenique P. Theory of surface plasmons and surface-plasmon polaritons. Rep Prog Phys 2007;70:1.

[21] Raza S, Toscano G, Jauho A-P, Wubs M, Mortensen NA. Unusual resonances in nanoplasmonic structures due to nonlocal response. Phys Rev B 2011;84:121412(R).

[22] Halevi P. Hydrodynamic model for the degenerate free-electron gas: generalization to arbitrary frequencies. Phys Rev $B$ 1995;51:7497-9.

[23] Toscano G, Raza S, Jauho A-P, Mortensen NA, Wubs M. Modified field enhancement and extinction by plasmonic nanowire dimers due to nonlocal response. Opt Express 2012;20:4176-88.

[24] Rodrigo SG, García-Vidal FJ, Martín-Moreno L. Influence of material properties on extraordinary optical transmission through hole arrays. Phys Rev B 2008;77:075401.

[25] Mortensen NA, Toscano G, Raza S, Stenger N, Yan W, Jauho A-P, Xiao S, Wubs M. Nanoplasmonics beyond Ohm's law. AIP Conf Proc 2012;1475:28-32.
[26] Mortensen NA. Nonlocal formalism for nanoplasmonics: phenomenological and semi-classical considerations. 2013; arXiv:1306.4651. DOI:10.1016/j.photonics.213.06.002.

[27] Hiremath KR, Zschiedrich L, Schmidt F. Numerical solution of nonlocal hydrodynamic Drude model for arbitrary shaped nano-plasmonic structures using Nédélec finite elements. J Comp Phys 2012;231:5890-6.

[28] Huang Q, Bao F, He S. Nonlocal effects in a hybrid plasmonic waveguide for nanoscale confinement. Opt Express 2013;21:1430-9.

[29] Nielsen RB, Fernandez-Cuesta I, Boltasseva A, Volkov VS, Bozhevolnyi SI, Klukowska A, Kristensen A. Channel plasmon polariton propagation in nanoimprinted V-groove waveguides. Opt Lett 2008;33:2800-2.

[30] Oulton RF, Bartal G, Pile DFP, Zhang X. Confinement and propagation characteristics of subwavelength plasmonic modes. New J Phys 2008;10: 105018.

[31] Esteban R, Borisov AG, Nordlander P, Aizpurua J. Bridging quantum and classical plasmonics with a quantum-corrected model. Nat Commun 2012;3:825.

[32] Ginzburg P, Hayat A, Berkovitch N, Orenstein M. Nonlocal ponderomotive nonlinearity in plasmonics. Opt Lett 2010;35:1551-3. 